\title{
The CHOICE (Choice of Health Options In prevention of Cardiovascular Events) replication trial: study protocol
} Lis Neubeck*1,2, Julie Redfern ${ }^{2,3}$, Tom Briffa ${ }^{4}$, Adrian Bauman ${ }^{5}$, David Hare ${ }^{6}$ and SB Freedman*1,2,7

Address: ${ }^{1}$ Faculty of Medicine, University of Sydney, Sydney, Australia, ${ }^{2}$ ANZAC Research Institute, Concord Hospital, Sydney, Australia, ${ }^{3}$ NHMRC NICS-Heart Foundation Fellow, Melbourne, Australia, ${ }^{4}$ School of Population Health, University of Western Australia, Perth, Australia, ${ }^{5}$ School of Public Health, University of Sydney, Sydney, Australia, ${ }^{6}$ Department of Cardiology, Austin Health, Department of Medicine, University of Melbourne, Melbourne, Australia and 7 Department of Cardiology, Concord Repatriation General Hospital, Sydney, Australia

Email: Lis Neubeck* - lneubeck@med.usyd.edu.au; Julie Redfern - julieredfern@dodo.com.au; Tom Briffa - tom.briffa@uwa.edu.au; Adrian Bauman - adrianb@health.usyd.edu.au; David Hare - David.HARE@austin.irg.au; SB Freedman* - ben@med.usyd.edu.au

* Corresponding authors

Published: 6 October 2008

BMC Cardiovascular Disorders 2008, 8:25 doi:10.1/86/147I-226I-8-25

This article is available from: http://www.biomedcentral.com/|47|-226I/8/25

(c) 2008 Neubeck et al; licensee BioMed Central Ltd.

This is an Open Access article distributed under the terms of the Creative Commons Attribution License (http://creativecommons.org/licenses/by/2.0), which permits unrestricted use, distribution, and reproduction in any medium, provided the original work is properly cited.
Received: 15 September 2008

Accepted: 6 October 2008

\begin{abstract}
Background: Although morbidity and mortality from coronary heart disease (CHD) are high, only a minority of acute coronary syndrome (ACS) survivors accesses an effective secondary prevention program. We aim to determine whether the previously proven CHOICE program can be replicated at multiple sites and whether ongoing reinforcement further improves risk factor modification.

Methods/design: Participants eligible for but not accessing standard cardiac rehabilitation will be randomly allocated to either a previously tested 3-month $\mathrm{CHOICE}$ program or a 30-month CHOICE program (CHOICE-plus). Both groups will participate in individualised risk factor modules of differing duration that involve choice, goal setting and telephone follow-up for three months. CHOICE-plus will also receive additional face-to-face and telephone reinforcement between three and 30 months. At one site we will recruit a randomised control group, receiving conventional care. Primary outcomes are lipid levels, blood pressure, physical activity levels and smoking rates. Secondary outcomes include readmission rates, death, the number of risk factors, other modifiable risk factors, quality of life and process evaluation measures over three years.
\end{abstract}

Discussion: We present the rationale and design of a multi-centre, replication study testing a modular approach for the secondary prevention of CHD following an ACS.

Trial Registration: [Clinical Trial Registration Number, ACTRNI 2608000 I82392]

\section{Background}

Coronary heart disease (CHD) is a leading cause of death and disability in Australia and costs associated with treatment are high [1]. Survivors of an acute coronary syndrome (ACS) have a $5 \%$ risk of a recurrence, some six times greater than that of the general population [2] and are advised to participate in a secondary prevention program $[3,4]$. Such programs incorporate lifestyle advice and pharmacotherapy to reduce recurrent cardiovascular events, improve survival and enhance quality of life [4]. 
The population is aging and thus the prevalence of CHD is expected to increase [5]. Similarly, the number of people surviving an ACS is rising largely due to improvements in acute treatment $[6,7]$. Because of these increases, the need to provide effective secondary prevention programs is escalating. In addition, three recent large studies concluded that effective risk factor reduction has reduced mortality from CVD by around 50\% [6-8].

Cardiac rehabilitation (CR) programs aim to reduce coronary risk factors, facilitate patients return to normal activities and reduce their overall cardiovascular risk, in addition to stabilising, slowing or even reversing the underlying atherosclerotic process $[9,10]$. However, despite the potential benefits of $\mathrm{CR}$, only a minority of people with CHD (10-30\%) participate in existing programs $[11,12]$. Reasons for poor attendance include geographical location, being of lower socio-economic group and low referral rates for the elderly and women [13]. In addition, even in those who attend, long-term compliance with exercise-only CR regimens is only around 50\% [13].

Disconcertingly, the majority of ACS survivors, who are at high risk of a future cardiac event, do not access a formal secondary prevention program resulting in a significant evidence-practice gap. In a single-centre study, it was demonstrated that ACS survivors not accessing CR had higher levels of individual risk factors including elevated total cholesterol (TC), low density lipoprotein (LDL) cholesterol, current smokers, hypertension, overweight, are less physically active, and were therefore at much greater overall risk than CR attendees [14]. Non-CR attendees also had much poorer knowledge of their risk factors, where $75 \%$ were unable to state even one of their own risk factors [14].

CHD is a chronic condition, and changes to lifestyle and adherence to pharmacotherapy have to be life-long. Studies have tried to address this evidence-practice gap through education and counselling of risk factor targets and medications with success $[15,16]$. Using a simple, innovative patient-centred modular approach to secondary prevention in a randomised controlled trial $(\mathrm{n}=208)$ $[17,18]$, it was found that the Choice of Health Options In prevention of Cardiovascular Events (CHOICE) program was readily acceptable to ACS survivors who did not access standard CR. It was also found that the intervention group significantly reduced individual cardiovascular risk factors and overall risk, and had significantly better risk factor knowledge at both three and 12 month follow-up compared to baseline and compared to having conventional care with their usual general practitioner and/or cardiologist [19-21]. Therefore, it was demonstrated at a single hospital site that the evidence-practice gap could be reduced for up to 12 months.
The original CHOICE trial provided a successful secondary prevention option for patients not accessing CR at a single site but it is now imperative to determine if the findings are replicable at multiple sites, delivered by multiple health professionals and to examine the processes which influence the implementation of a successful trial into a "real-life" setting [22]. The aim of this study is to establish if the CHOICE program can be replicated at multiple hospitals over three years. We also aim to investigate whether 30 months of telephone support is more beneficial than three months.

\section{Methods/design \\ Design}

This replication study, with a single site randomised control group, with three year follow up will be conducted at four tertiary referral hospitals in Sydney, Australia (figure 1 ) and is registered on the Australian and New Zealand Clinical Trials Registry (ACTRN12608000182392). Across all four sites patients not accessing standard CR will be randomly allocated to either a group participating in a three month CHOICE program or a 30 month CHOICE program (CHOICE-plus). At one site, patients not accessing $\mathrm{CR}$ will be allocated to one of three groups; the previously described intervention groups or a randomised control group participating in conventional care (figure 1 ), which will allow investigation of the effect of an additional nutrition module and a more tailored approach to depression.

Blinded assessments will be conducted at baseline and 36 months during a face-to-face interview. At 12 months, assessments will be completed by the intervention nurse and to ensure validity, a sample of 20 patients will have their measurements repeated by an independent assessor, blinded to group allocation. At follow-up assessments, additional data will be collected, including details of any unplanned hospital admissions, medications and doses and details of visits to the family physician, cardiologist and attendance at any community program pertaining to their heart health. Ethical approval for this study has been granted by Sydney South West Area Health Services and will be sought from the University of Sydney Human Research Ethics Committees. Written and informed consent will be obtained from all study participants prior to commencement.

\section{Study population}

The hospital CR coordinators who review the Cardiology Department admission summaries daily will identify potential participants. Inclusion criteria for the study are; diagnosis of ACS up to eight weeks prior to recruitment; refusal of the initial invitation to participate in standard $\mathrm{CR}$; failure to attend initial CR assessment. Exclusion criteria are: clinical diagnosis of uncompensated, severe car- 


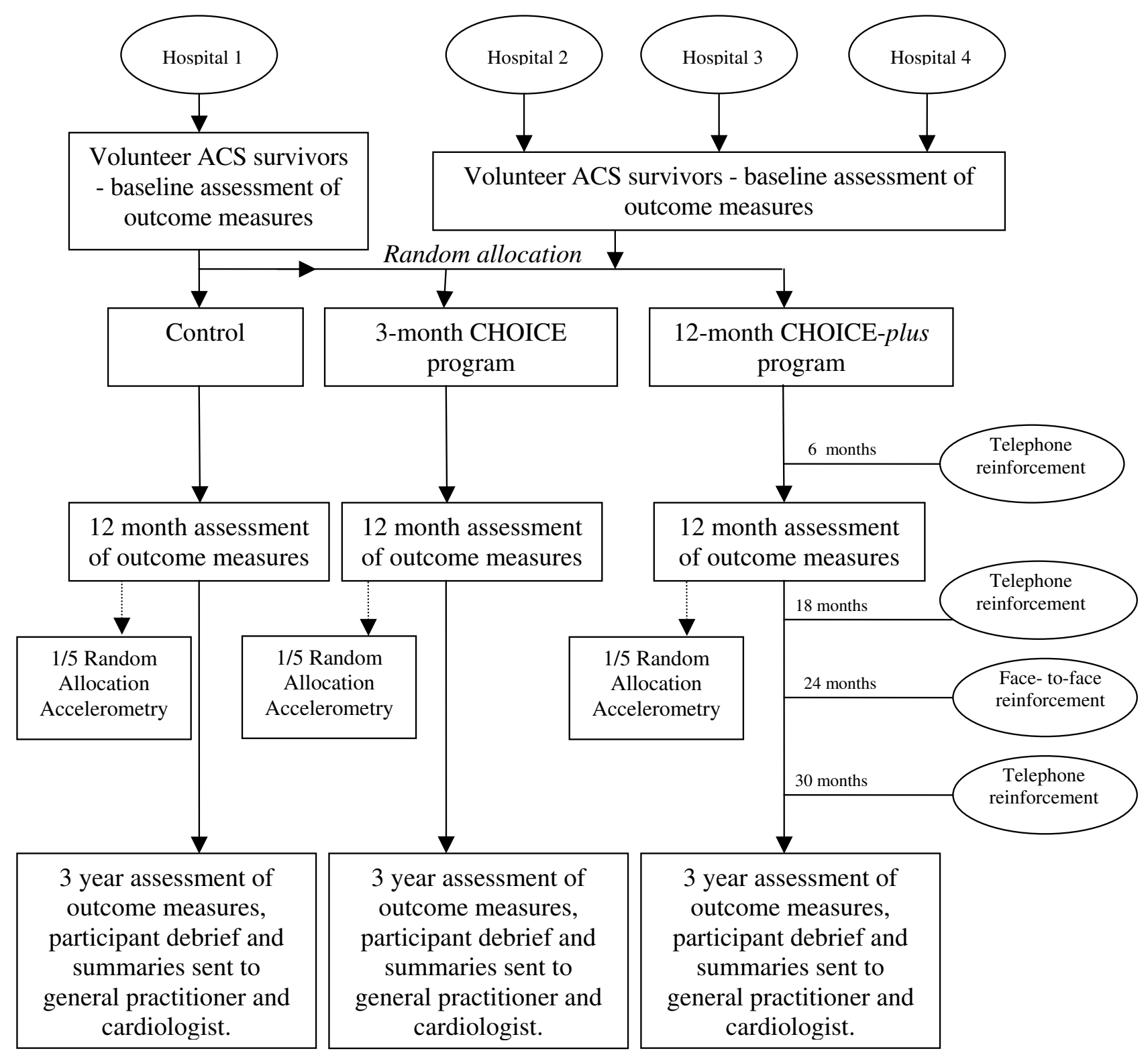

Figure I

Design of the CHOICE study. ACS: acute coronary syndrome. CHOICE: Choice of Health Options In prevention of Cardiovascular Events.

diac failure (Class IV); uncontrolled arrhythmia or angina; severe or symptomatic aortic stenosis; persistent hypotension ( $\mathrm{SBP}<90 \mathrm{mmHg}$ ); clinical diagnosis of a severe coexisting medical condition that would prevent participation (e.g., dementia, a terminal illness, severe rheumatoid arthritis); or insufficient English to provide written informed consent. The primary criterion for eligibility for recruitment will be declining an offer to access existing CR. This will also include patients who are not offered CR while an inpatient but decline a subsequent offer to attend the CR program.

\section{Group allocation}

Patients meeting the inclusion and exclusion criteria will be approached by the hospital CR coordinator or research assistant, during their inpatient stay where possible, or via letter or telephone soon after hospital discharge. Those who volunteer will have a baseline assessment and an initial face-to-face module selection and goal-setting session (approximately one hour in duration). This session will take place during the inpatient stay or in outpatient clinical consulting rooms, or, if necessary, at the participant's home, within one month of discharge. After baseline 
measurements are completed, at three sites, participants will be randomly allocated to one of the two groups (CHOICE or CHOICE-plus). At the remaining site participants will be randomly allocated to one of three groups (control, CHOICE or CHOICE-plus). Randomisation will be undertaken by an independent researcher (at the University of Sydney) with a computer-generated random allocation sequence and will be concealed from the health professional obtaining consent and conducting baseline assessment.

\section{Interventions}

Participants randomly allocated to the control group at the single site will participate in ongoing conventional care, aimed at managing their cardiovascular health as directed by their family physician, ideally in consultation with their cardiologist. The control group will be advised to seek the advice of their family physician and cardiologist but will receive no additional intervention as a result of participation in the study.

Participants in both the CHOICE and CHOICE-plus groups will take part in a three-month patient-centred modular secondary prevention program. The CHOICEplus group will also be offered additional face-to-face reinforcement at 12 and additional telephone reinforcement at six, 18 and 30 months.

As described elsewhere, the CHOICE intervention includes a one-hour initial consultation and follow-up phone calls over three months [20]. The program is designed to have an individualised, structured, case-management approach and is overseen by treating physicians. The development and implementation of the CHOICE program can be divided into four stages (figure 2). Stage one involves the development of modules and tailoring of leaflets to access recommended local services [21]. Stage two is a face-to-face risk factor assessment, lasting approx- imately one hour in which the patient is assisted to generate a list of their own relevant risk factors. In stage three, patients make guided choices about which risk factors they will address, participate in realistic goal-setting informed by national targets (table 1) [9] and then select management option(s) for lowering risk. The relevant patient information leaflets, described in Stage 1, support these choices. All CHOICE patients participate in the core module for lowering cholesterol as well as up to two choice modules from blood pressure (BP)-lowering, smoking cessation, increased physical activity and nutrition. Patients will have the option to add additional modules on completion of the three-month program. Additional module information will be given over the telephone and written information will be mailed to the patient. Stage four, is telephone follow-up, consisting of approximately four calls of around 10 minutes duration over a three-month period, during which each patient's risk factor(s) goals and strategies are re-evaluated and mutually changed if necessary. CHOICE-plus patients will receive additional telephone follow-up over 3 years as previously outlined (figure 1).

\section{Outcome measures}

The primary outcomes are $\mathrm{TC}$, systolic blood pressure (SBP), smoking rates and physical activity levels at 12 and 36 months (Table 2). Secondary outcomes include readmission rates, all-cause mortality, cardiac mortality, number of cardiac risk factors, waist circumference and process measures to assess barriers to implementation and fidelity to the intervention components (Table 2).

Self-reported physical activity will be validated in a onefifth subset of the cohort at 12 months by using accelerometers. Accelerometry is now considered the preferred method of objectively measuring physical activity as it provides data that allows individual examination of ambulatory activity frequency, intensity and duration

Table I: Risk factor targets

\begin{tabular}{|c|c|}
\hline Risk factor & National Heart Foundation of Australia targets [9] \\
\hline Smoking & Complete cessation \\
\hline Nutrition & Establish and maintain healthy eating \\
\hline Alcohol & Low risk alcohol consumption \\
\hline Physical activity & 30 minutes of moderate activity on most days of the week \\
\hline \multirow[t]{3}{*}{ Healthy weight } & Waist measurement \\
\hline & Male $\leq 94 \mathrm{~cm}$ Female $\leq 80 \mathrm{~cm}$ \\
\hline & BMI $18.5-24.9 \mathrm{~kg} / \mathrm{m}^{2}$ \\
\hline \multirow[t]{3}{*}{ Lipids } & $\mathrm{LDL}<2.0 \mathrm{mmol} / \mathrm{l}$ \\
\hline & $\mathrm{HDL}>1.0 \mathrm{mmol} / \mathrm{l}$ \\
\hline & Triglycerides $<1.5 \mathrm{mmol} / \mathrm{l}$ \\
\hline \multirow[t]{3}{*}{ Blood pressure } & Adults $\geq 65$ (unless they have diabetes and/or renal insufficiency and/or proteinuria $\geq 0.25 \mathrm{~g} / \mathrm{day}$ ) $<140 / 90 \mathrm{mmHg}$ \\
\hline & Adults $<65$ years; adults with diabetes and/or renal insufficiency and/or proteinuria $0.25-1.0 \mathrm{~g} / \mathrm{day}<130 / 80 \mathrm{mmHg}$ \\
\hline & Adults with proteinuria $>1 \mathrm{~g} /$ day $<125 / 75 \mathrm{mmHg}$ \\
\hline Diabetes & Identify those with previously undiagnosed type 2 diabetes. In those with diabetes maintain $\mathrm{HbAIC} \leq 7.0 \%$ \\
\hline
\end{tabular}




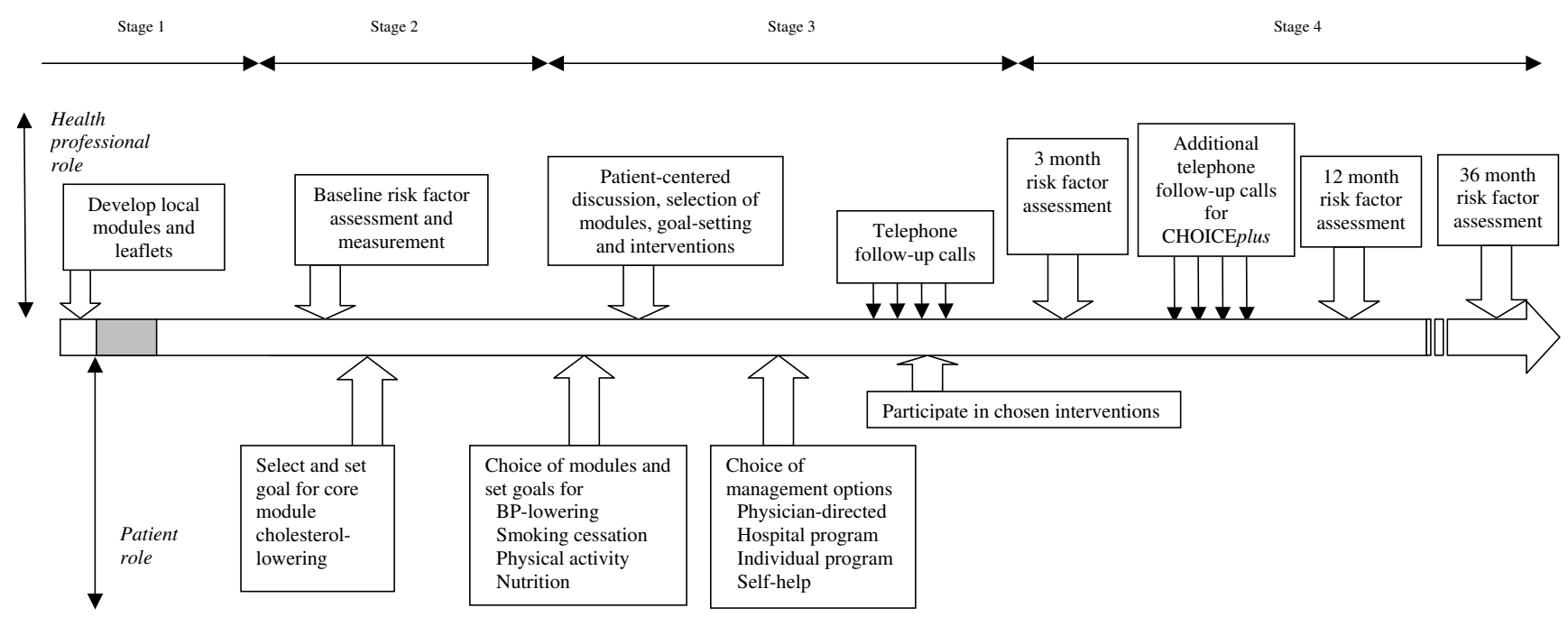

\section{Figure 2}

Model of CHOICE (Choice of Health Options In prevention of Cardiovascular Events) program after acute coronary syndrome (ACS).

$[23,24]$. For this trial an Actigraph GT1M (formerly Computer Science and Applications monitor), the most widely accepted accelerometer in research, will be utilised [25]. The matchbox size accelerometer will be attached to a belt that the participants in both the intervention and control groups will be asked to wear for a period of seven consecutive days at one year and three years. The Active Australia survey [26] records information from the seven-day period immediately prior to the survey, and therefore will be completed at the end of the week in which the acceler- ometer is worn. Thus the accelerometry data and Active Australia will record the same seven-day period.

To evaluate the generalisability of the previously proven CHOICE study [21], process measures of the intervention will include: record of participant recruitment, withdrawal from the program, the context of the interventions and the resources used. Barriers to implementation will be documented. Each participant will also answer questionnaires, which will determine the dose and exposure to the

Table 2: Trial end points

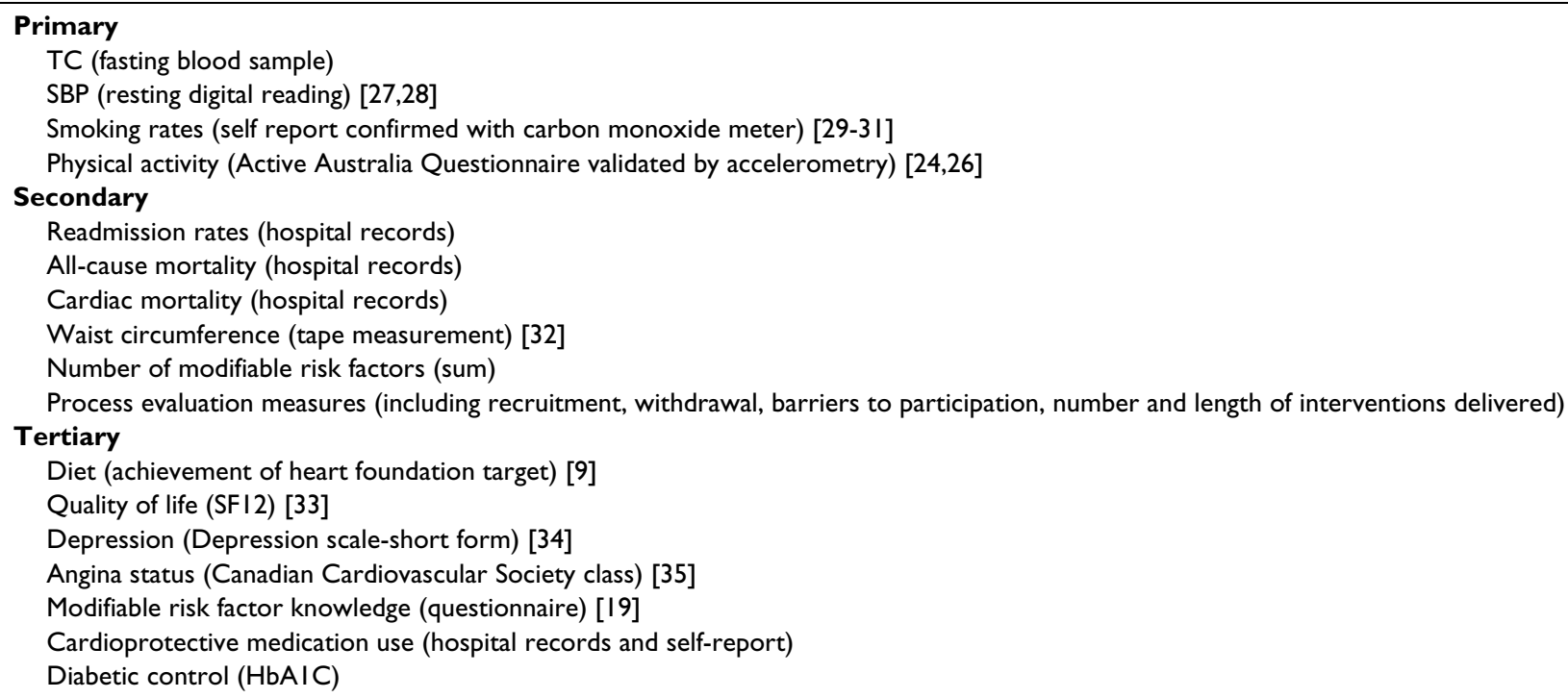


elements of the program. Through this we will examine fidelity of the program to the intervention components.

\section{Sample size}

Given the compelling evidence that lowering TC lowers cardiovascular events, our sample size is calculated based on change in TC. In the previous study with 70/group, we found a 25.5 (SD 1.0$) \mathrm{mg} / \mathrm{dL}(0.66 \mathrm{mmol} / \mathrm{L})$ greater reduction in TC at 12 months in the CHOICE than control group. To demonstrate a difference between CHOICE and CHOICE-plus groups, 138 per group in the RCT will give $80 \%$ power $(2$-tailed, $\mathrm{P}<0.05)$ to detect a $0.35 \mathrm{SD}$ effect $(13.5 \mathrm{mg} / \mathrm{dl}(0.35 \mathrm{mmol} / \mathrm{L})$ difference $)$ in TC at 12 months or 3 years. To demonstrate a difference between the control and intervention groups at the single randomized site, 64 patients will be needed in the control group for $80 \%$ power. To allow for $20 \%$ loss to follow-up and a design effect of up to 1.15 (ICC 0.05) due to cluster sampling, a total of 400 patients will be recruited across the four sites.

\section{Statistical analysis}

Primary analyses will be conducted by intention-to-treat using SPSS for Windows (Version 12.01) and will be presented as mean and standard error of the mean or proportions. Differences in outcome measures, between and within groups, will be compared using repeated measures ANOVAs for continuous variables and either $\chi^{2}$ tests or Fishers exact tests, as appropriate, for proportions of categorical variables. Two tailed $p$ values of $<0.05$ will be considered significant. Number needed to treat will also be calculated to estimate the number of patients who need to receive the CHOICE intervention to lower each of the major risk factors (TC, LDL-cholesterol, BP, smoking cessation, physical activity and overweight) to below current national targets.

\section{Discussion}

The findings of this replication study could have wideranging implications for the management of patients with CHD who are at highest risk of adverse events, namely survivors of an ACS. We anticipate this study will demonstrate that the large evidence-practice gap for the many ACS survivors not accessing CR can be narrowed by a brief, flexible and tailored modular intervention (CHOICE) to change behavior, reduce overall cardiac risk, and thereby reduce the likelihood of death, infarction, and recurrent symptoms. By demonstrating the generalisability of the previously proven CHOICE program, this approach, using existing personnel and community resources could easily be adopted state-wide at relatively low cost.

The ultimate goal for our patients is to maintain behavioural change and risk factor reduction in the long term within their local community. At the conclusion of this study we anticipate that we will be able to determine whether ongoing reinforcement is important to lower risk, and if this strategy can be implemented at multiple sites and consequently narrow the evidence-practice gap in the longer term.

\section{Competing interests}

The authors declare that they have no competing interests.

\section{Authors' contributions}

LN drafted the manuscript and assisted with revision of study design and co-ordination. JR conceived of the study, design and co-ordination and helped to draft the manuscript. $\mathrm{TB}, \mathrm{AB}, \mathrm{DH}$ and $\mathrm{BF}$ were responsible for the original study design and review of the manuscript. All authors read and agree to the manuscript as written.

\section{Acknowledgements}

We thank HCF Health and Medical Research Foundation for funding towards the design and implementation of this study

Dr Julie Redfern is a National Institute of Clinical Studies-Heart Foundation Fellow and we thank this organisation for their support. NICS is an institute of the National Health and Medical Research Council (NHMRC), Australia's leading funding body for health and medical research.

\section{References}

I. Australian Institute of Health and Welfare: Australia's Health 2006. AlHW Cat No AUS 732006.

2. World Health Organization: Secondary prevention of noncommunicable diseases in low- and middle-income countries through community-based and health service interventions. Geneva: WHO; 2002.

3. Aroney C, Aylward P, Kelly A, Chew D, Clune E: Guidelines for the management of acute coronary syndromes 2006. Med J Aust 2006, I 84(8):s3-s30.

4. McAlister FA, Lawson FME, Teo KK, Armstrong PW: Randomised trials of secondary prevention programs in coronary heart disease: systematic review. Br Med J 200I, 323(73 I 9):957-962.

5. Australian Institute of Health and Welfare: Heart, stroke and vascular diseases - Australian facts 2004. AlHW Cat No CVD 27 2004.

6. Unal B, Critchley JA, Capewell S: Explaining the Decline in Coronary Heart Disease Mortality in England and Wales Between I 981 and 2000. Circulation 2004, I 09(9): I I0I-I I 07.

7. Ford ES, Ajani UA, Croft JB, Critchley JA, Labarthe DR, Kottke TE, Giles WH, Capewell S: Explaining the Decrease in U.S. Deaths from Coronary Disease, 1980-2000. N Engl J Med 2007, 356(23):2388-2398.

8. Taylor R, Dobson A, Mirzaei M: Contribution of changes in risk factors to the decline of coronary heart disease mortality in Australia over three decades. Eur J Cardiovasc Prev Rehabil 2007, I3(5):760-768.

9. National Heart Foundation of Australia and the Cardiac Society of Australia and New Zealand: Reducing risk in heart disease. National Heart Foundation of Australia; 2007.

10. AHA Scientific Statement: Cardiac rehabilitation and secondary prevention of coronary heart disease. Circulation 2005, I I I (3):369-376.

II. Scott IA, Lindsay KA, Harden HE: Utilisation of outpatient cardiac rehabilitation in Queensland. Med J Aust 2003, I 79:34I-345.

12. Suaya JA, Shepard DS, Normand S-LT, Ades PA, Prottas J, Stason WB: Use of Cardiac Rehabilitation by Medicare Beneficiaries After Myocardial Infarction or Coronary Bypass Surgery. Circulation 2007, I I 6( I 5): | 653- I662. 
13. Ades PA: Cardiac Rehabilitation and Secondary Prevention of Coronary Heart Disease. N Engl J Med 200I, 345(I 2):892-902.

14. Redfern J, Ellis ER, Briffa T, Freedman SB: High risk-factor level and low risk-factor knowledge in patients not accessing cardiac rehabilitation after acute coronary syndrome. Med J Aust 2007, I 86(I):2I-25

15. DeBusk RF, Miller NH, Superko HR, Dennis CA, Thomas RJ, Lew HT, Berger WE, Heller RS, Rompf J, Gee D, Kraemer HC, Bandura A, Ghandour G, Clark M, Shah RV, Fisher L, Taylor CB: A case-management system for coronary risk factor modification after acute myocardial infarction. Ann Intern Med 1994 | 20(9):72|-729.

16. Haskell W, Alderman E, Fair J, Maron D, Mackey S, Superko H, Williams $\mathrm{P}$, Johnstone I, Champagne M, Krauss RM: Effects of multiple risk factor reduction on coronary atherosclerosis and clinica cardiac events in men and women with coronary artery disease, the Stanford coronary risk intervention project (SCRIP). Circulation 1994, 89:975-990.

17. Redfern J, Ellis E, Briffa T, Freedman SB: Modular prevention of heart disease following acute coronary syndrome (ACS) [ISRCTN42984084]. BMC Cardiovasc Disord 2006, 6(I):26.

18. Redfern J, Ellis E, Briffa T, Freedman S: Development and testing of innovative patient resources for the management of coronary heart disease (CHD): a descriptive study. BMC Health Serv Res 2006, 6(95):

19. Redfern J, Ellis E, Briffa T, Freedman S: Modular secondary prevention based on patient choice improves risk factor profile and knowledge after acute coronary syndrome: $A$ randomized controlled trial. J Am Coll Cardiol 2007, 49(9):347A.

20. Redfern J, Briffa T, Ellis E, Freedman S: Patient-centered modular secondary prevention following acute coronary syndrome: $A$ randomized controlled trial. J Cardiopulm Rehabil Prev 2008.

21. Redfern J, Briffa T, Ellis E, Freedman S: Choice of secondary prevention improves risk factors after acute coronary syndrome: one year follow-up of the CHOICE (Choice of Health Options In prevention of Cardiovascular Events) randomised controlled trial. Heart 2008.

22. Nutbeam D, Bauman A: Evaluation in a nutshell: A practical guide to the evaluation of health promotion programs. Sydney: McGraw Hill Medical; 2006.

23. Brown WJ, Trost SG, Bauman A, Mummery K, Owen N: Test-retest reliability of four physical activity measures used in population surveys. J Sci Med Sport 2004, 7(2):205-2I5.

24. Ward D, Evenson K, Vaughn A, Rodgers A, Troiano R: Accelerometer use in physical activity: best practices and research recommendations. Med Sci Sports Exerc 2005, 37(I I Suppl):S582-S585

25. Welk G, (ed): Use of accelerometry-based activity monitors to assess physical activity. In Physical activity assessments for healthrelated research Champaign: Human Kinetics Publishers; 2002.

26. Australian Institute of Health and Welfare: The active Australia survey: A guide for implementation, analysis and reporting. In Cat no CVD 22 Canberra: AlHW; 2003.

27. Omron instruction manual: Automatic blood pressure monitor with arm cuff. Model IA2.

28. O'Brien E, Asmar R, Beilin L, Imai Y, Mallion J-M, Mancia G, Mengden T, Myers M, Padfield P, Palatini P, Parati G, Pickering T, Redon J, Staessen J, Stergiou G, Verdecchia P, on behalf of the European Society of Hypertension Working Group on Blood Pressure Monitoring: European Society of Hypertension recommendations for conventional, ambulatory and home blood pressure measurement. J Hypertens 2003, $21: 821-848$.

29. Cunnington AJ, Hormbrey P: Breath analysis to detect recent exposure to carbon monoxide. Postgrad Med J 2002, 78(9 | 8):233-237

30. Pearce MS, Hayes $L$, on behalf of the Newcastle Heart Project and the Newcastle Thousand Families S: Self-Reported Smoking Status and Exhaled Carbon Monoxide: Results From Two Population-Based Epidemiologic Studies in the North of England. Chest 2005, I 28(3): 1233-1238.

31. Fagerström K: Time to first cigarette; the best single indicator of tobacco dependence? Monaldi Arch Chest Dis 2003 59(I):91-94.

32. Lean MEJ, Han TS, Morrison CE: Waist circumference as a measure for indicating need for weight management. BM] 1995 , 31 I(6998): |58-16I.
33. Ware J, Kosinski M, Keller S: A I 2-Item Short-Form Health Survey: Construction of scales and preliminary tests of reliability and validity. Med Care 1996, 34(3):220-233.

34. Shi W, Wu N, Stewart A, Toia D, Hare D: Depression scale-short form: validation of a new 60 second depression screening tool for cardiac patients. Heart Lung Circ 2008, I7(s3):s24.

35. Campeau LMD: Grading of Angina Pectoris. Circulation 1976 54(3):522-523.

\section{Pre-publication history}

The pre-publication history for this paper can be accessed here:

http://www.biomedcentral.com/1471-2261/8/25/prepub
Publish with Bio Med Central and every scientist can read your work free of charge

"BioMed Central will be the most significant development for disseminating the results of biomedical research in our lifetime. "

Sir Paul Nurse, Cancer Research UK

Your research papers will be:

- available free of charge to the entire biomedical community

- peer reviewed and published immediately upon acceptance

- cited in PubMed and archived on PubMed Central

- yours - you keep the copyright
BioMedcentral 